day 1,5 , and 10 , and after 40 weeks adjusted for the gestational age in preterm LBW infants. Blood pressure was measured at each visit using Welch Allyn VSMTM 300 monitor.

Results At birth, the NBW infants had significantly lower BCD (difference -9.3 cap/area, 95\% CI: -1.5 to $-17.1, \mathrm{p}=0.021$ ) and MCD (difference -12.6 cap/area, 95\% CI: -1.5 to $-21.7, p=0.025)$ compared to the LBW infants. LBW oxygen group had a significantly lower SBP (mean difference -9.5 $\mathrm{mmHg}, 95 \% \mathrm{CI}:-1$ to $-19, \mathrm{p}=0.047$ ), DBP (difference -13 $\mathrm{mmHg}, 95 \% \mathrm{CI}:-4$ to $-22, \mathrm{p}=0.009)$. At 40 weeks old, the LBW oxygen group showed a significant reduction in BCD (difference -19.3 cap/area, 95\% CI: -9 to $-30, p=0.003$ ) and MCD (difference -22 cap/area, 95\% CI: -8 to -36 $\mathrm{p}=0.007)$. Similarly the LBW non-oxygen group had a significant reduction in BCD (difference -29 cap/area, 95\% CI -17 to $-41 \mathrm{p}<0.0001$ ) and MCD (mean difference -29 cap/area, $95 \% \mathrm{CI},-16$ to $-41 \mathrm{p}<0.001)$. Both $\mathrm{LBW}$ groups showed a significant rise in BP. The rise in SBP (mean difference $24 \mathrm{mmHg}$, 95\% CI: 14-34, p<0.0001) and DBP (mean difference $14 \mathrm{mmHg}, 95 \% \mathrm{CI}: 7-22, \mathrm{p}<0.001$ ) was more pronounced in LBW oxygen group compared to the LBW control group (difference $14 \mathrm{mmHg}, 95 \% \mathrm{CI}$ : 0.5-27, $\mathrm{p}=0.043$ and difference $9 \mathrm{mmHg}, 0.3-19, \mathrm{p}=0.056$ respectively)

Conclusions We confirm that LBW infants have higher capillary density at birth but develop significant capillary rarefaction and increase in their blood pressure at 40 weeks compared to NBW infants. Oxygen therapy in the neonatal period in LBW infants was associated with higher blood pressure levels but we could not detect any effect on capillary rarefaction. Further studies are needed to investigate the humoral factors that trigger the changes of microcirculation in LBW infants during the neonatal period which may be of importance in preventing hypertension in later life.

\section{EVOLUTION OF EUROPEAN SOCIETY OF CARDIOLOGY GUIDELINES SINCE 2000: A SYSTEMATIC APPRAISAL}

${ }^{1}$ Charles Badu-Boateng*, ${ }^{2}$ Arash Yavari, ${ }^{3}$ Rizwan Sarwar. 'King's College London, GKT School of Medicine, London, UK; ${ }^{2}$ ohn Radcliffe Hospital, Cardiology Department, Oxford, UK; ${ }^{3}$ John Radcliffe Hospital, Cardiology Department, Oxford, UK

\subsection{6/heartjnl-2017-311726.101}

Background Guidelines from the European Society of Cardiology (ESC) summarise the best available evidence in the management of cardiac disease. These guidelines are graded according to ESC-predefined scales of class of recommendation from I-III and levels of evidence from A-C. We evaluated the development of ESC guidelines since 2000.

Method We reviewed recommendations from all ESC guidelines from 2000-14, collating classes of recommendation and level of evidence for each. We assessed the number and percentages of each class of recommendation and determined temporal changes in proportion.

Results Out of 52 guidelines, we extracted 4547 recommendations, with $67 \%$ being in Class I or III, indicating unequivocal guidance. Regarding the level of evidence for these recommendations, we found that only $18 \%$ were class A, indicating high quality evidence, and 50\% were class C, indicating consensus opinion or small studies. From 2000-2014, there were minor changes in the proportions of different classes of recommendation and levels of evidence.

Conclusions Medicine is rapidly changing and cardiologists face difficult challenges in the application of a growing number of recommendations of difference evidence level to guide clinical practice. Our analysis highlights that 33\% of all guideline statements are in the equivocal class (II), with $82 \%$ of guidelines not based upon the highest level of evidence. Over 14 years there have been minimal changes in proportions of class of recommendation and level of evidence. Our findings suggest the need to improve the level of evidence underpinning current guidance to increase the proportion of unequivocal guidance recommendations.

\section{THE ABILITY OF CARDIOGONIOGMETRY COMPARED TO FLOW FRACTIONAL RESERVE AT IDENTIFYING PHYSIOLOGICALLY SIGNIFICANT CORONARY STENOSIS: THE CARDIOFLOW STUDY}

1,Oliver I Brown*, ${ }^{2}$ Andrew L Clark, ${ }^{2}$ Raj Chelliah, ${ }^{2}$ Benjamin Davison, ${ }^{2}$ Adam N Mather, ${ }^{2}$ Michael S Cunnington, ${ }^{2}$ Joseph John, ${ }^{2}$ Albert Alahmar, ${ }^{2}$ Richard Oliver, ${ }^{2}$ Konstantinos Aznaouridis, ${ }^{2}$ Angela Hoye. ${ }^{1}$ Hull York Medical School; ${ }^{2}$ Hull and East Yorkshire Hospital NHS trust

\subsection{6/heartjnl-2017-311726.102}

Introduction Cardiogoniometry (CGM) is method of 3-dimensional electrocardiographic assessment which has been previously shown to identify patients with angiographically defined, stable coronary artery disease (CAD). However, angiographic evidence of $\mathrm{CAD}$, does not always correlate to physiologically significant CAD. The aim of our study was to assess the ability of CGM to detect physiologically significant coronary stenosis defined by fractional flow reserve (FFR).

Methods In a tertiary cardiology centre, patients with single vessel CAD were enrolled into a prospective double blinded observational study. A baseline CGM recording was performed at rest. A second CGM recording was then performed during the FFR procedure, at the time of maximal hyperaemia. A significant CGM result was defined as an automatically calculated ischaemia score $<0$ and a significant FFR ratio defined as $<0.8$. After enrolment, CGM and FFR results were compared and markers of diagnostic performance (sensitivity, specificity, positive predictive value and negative predictive value) were calculated at rest and during maximal hyperaemia. Statistical agreement between CGM and FFR was calculated by the Kappa statistic.

Results Forty patients were included (aged 61.1土11.0; 60.0\% male), of which sixteen (40\%) were found to have significant CAD when assessed by FFR. Markers of diagnostic performance of CGM are shown in the table.

Conclusion The diagnostic performance of CGM to detect physiologically significant stable CAD is poor at rest. Although, the diagnostic performance of CGM improves substantially during maximal hyperaemia, it does not reach sufficient levels of accuracy to be used routinely in clinical practice.

\begin{tabular}{lll} 
Abstract $\mathbf{1 0 3}$ Table 1 & & \\
\hline & CGM at rest $(\mathrm{n}=\mathbf{4 0})$ & $\begin{array}{l}\text { CGM during maximal } \\
\text { hyperaemia }(\mathrm{n}=40)\end{array}$ \\
\hline Sensitivity & $31.3 \%$ & $68.8 \%$ \\
Specificity & $62.5 \%$ & $54.2 \%$ \\
Positive predictive value & $35.7 \%$ & $50.0 \%$ \\
Negative predictive value & $57.7 \%$ & $72.2 \%$ \\
Kappa statistic for agreement & $-0.06, \mathrm{p}=0.64$ & $0.21, \mathrm{p}=0.15$ \\
\hline
\end{tabular}

